\title{
Desktop Electrospinning
}

\section{A Single Extruder 3D Printer for Producing Rigid Plastic and Electrospun Textiles}

\author{
Michael L. Rivera \\ Human-Computer Interaction Institute \\ Carnegie Mellon University \\ Pittsburgh, PA \\ mlrivera@cs.cmu.edu
}

\author{
Scott E. Hudson \\ Human-Computer Interaction Institute \\ Carnegie Mellon University \\ Pittsburgh, PA \\ scott.hudson@cs.cmu.edu
}

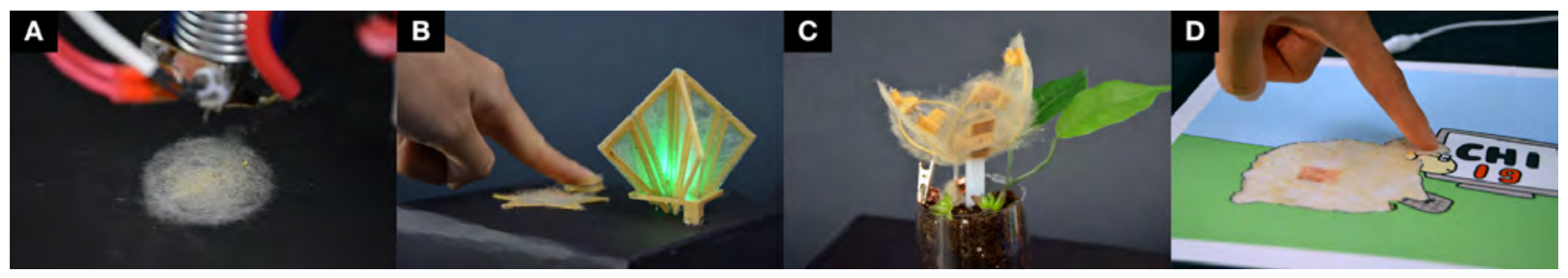

Figure 1: A range of objects fabricated on our novel 3D printer using rigid plastic and electrospun textiles: (A) a close-up of our printer electrospinning; (B) an origami-style folding lamp with piezoresistve brightness control and a soft custom-shaped capacitive toggle switch that use electrospun textiles for sensing and tactile experiences; (C) an actuated electrospun and rigid plastic flower that opens when an electrospun textile liquid sensor detects sufficient water in the soil; (D) a sheep comic that uses electrospun textile with capacitive sensing to create an interactive tactile experience.

\begin{abstract}
We present a new type of 3D printer that combines rigid plastic printing with melt electrospinning- a technique that uses electrostatic forces to create thin fibers from a molten polymer. Our printer enables custom-shaped textile sheets (similar in feel to wool felt) to be produced alongside rigid plastic using a single material (i.e. PLA) in a single process. We contribute open-source firmware, hardware specifications, and printing parameters to achieve melt electrospinning. Our approach offers new opportunities for fabricating interactive objects and sensors that blend the flexibility, absorbency and softness of produced electrospun textiles with the structure and rigidity of hard plastic for actuation, sensing, and tactile experiences.
\end{abstract}

\footnotetext{
Permission to make digital or hard copies of all or part of this work for personal or classroom use is granted without fee provided that copies are not made or distributed for profit or commercial advantage and that copies bear this notice and the full citation on the first page. Copyrights for components of this work owned by others than the author(s) must be honored. Abstracting with credit is permitted. To copy otherwise, or republish, to post on servers or to redistribute to lists, requires prior specific permission and/or a fee. Request permissions from permissions@acm.org. CHI 2019, May 4-9, 2019, Glasgow, Scotland UK

() 2019 Copyright held by the owner/author(s). Publication rights licensed to ACM.

ACM ISBN $978-1-4503-5970-2 / 19 / 05 \ldots \$ 15.00$

https://doi.org/10.1145/3290605.3300434
}

\section{CCS CONCEPTS}

- Human-centered computing $\rightarrow$ Human computer interaction $(\mathrm{HCI}) ; \bullet$ Hardware $\rightarrow$ Electromechanical systems;

\section{KEYWORDS}

3D Printing, Melt Electrospinning, Soft Material Fabrication, Textiles

\section{ACM Reference Format:}

Michael L. Rivera and Scott E. Hudson. 2019. Desktop Electrospinning: A Single Extruder 3D Printer for Producing Rigid Plastic and Electrospun Textiles. In CHI Conference on Human Factors in Computing Systems Proceedings (CHI 2019), May 4-9, 2019, Glasgow, Scotland UK. ACM, New York, NY, USA, 12 pages. https: //doi.org/10.1145/3290605.3300434

\section{INTRODUCTION}

Additive manufacturing, specifically 3D printing, has gained considerable attention in both consumer and research domains over the last decade. Until recently, the technology has been primarily used to create functional rigid objects. The addition of flexible filaments and other pliable printable materials (e.g., silicone) has opened up new opportunities for fabricating objects that blend rigidity and compliance. In a similar fashion, researchers in the field of Human-Computer Interaction (HCI) have explored how to 3D print soft interactive objects using textiles, ranging from fabric $[25,29]$ to felted yarn [9]. 
The present work continues to explore the boundary between rigidity and softness but uses a new material production approach to embed the fabrication of textiles directly into a consumer-grade desktop 3D printing process. Our approach supports both rigid plastic printing and melt electrospinning-a technique that uses an electrostatic forces to produce thin fibers from a molten polymer (e.g., PLA, a commonly used 3D printing filament). Controlling the deposition of these fibers enables us to produce custom-shaped textile sheets that feel similar to wool felt alongside rigid plastic, opening up a range of new opportunities for fabricating interactive objects and sensors (Figure 1).

We view this work as a step towards a future where material science and HCI blend, pushing the boundary of what and how interactive objects can be made using consumergrade digital fabrication technology. To our knowledge, we are the first to use melt electrospinning in tandem with 3D printing to create interactive objects. We make the following contributions:

- We present a novel approach to fabricating combined rigid plastic and textile-based objects.

- We demonstrate a new type of consumer-grade 3D printer that combines melt electrospinning and rigid plastic printing using a single extruder.

- We contribute open-source firmware, hardware specifications, and printing parameters to achieve melt electrospinning on consumer-grade 3D printers.

- Lastly, we show a series of applications and sensing capabilities enabled by using our approach.

\section{RELATED WORK}

Our work relates to several areas of research including soft material fabrication, textiles in the domain of 3D printing, and electrospinning. In this section, we summarize key work in these areas and describe how our approach differs.

\section{Soft Material Fabrication}

In recent years, researchers have explored how to use the flexibility and softness of materials to create interactive objects. Some work creates sensors by mixing soft and conductive materials together such as coating sponges in conductive ink [22], curing a carbon-filled elastomer [37], embedding conductive ink into 3D printing with flexible thermoplastic polyurethane (TPU) [1], and printing TPU and conductive filaments together [31]. Using the same flexible material of TPU, Ion et al. print mechanisms composed of metamaterial cells [12]. Similarly, Megaro et al. demonstrate an optimization-based design tool for creating compliant mechanisms via 3D printing of a flexible photopolymer [20]. Others have shown how computational design of an object's structure can control its flexibility either when printed in

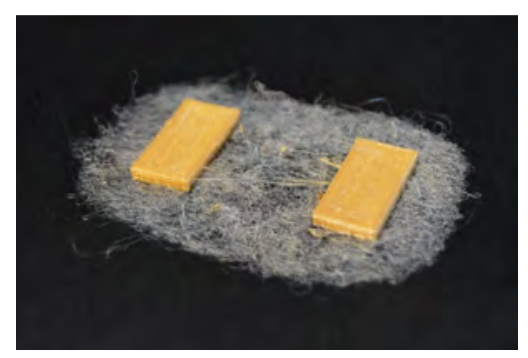

Figure 2: A simple hinge fabricated with our 3D printer using rigid plastic printing and melt electrospinning on the same extruder.

soft materials [20,38] or rigid materials [3, 19, 26, 32]. Lastly, some work has developed new processes for creating soft materials such as a liquid printer that fabricates flexible objects within a vat of gel $[6,7]$, and manufacturing techniques that make conductive textiles for wearable computing [27].

Much like these prior efforts, our work demonstrates a way to fabricate objects with soft materials. However, we focus on fabricating electrospun textiles and rigid plastic together in the same printing process as seen in Figure 2.

\section{Smart Textiles}

Another related line of work in the HCI community has explored the fabrication and design of "smart" textiles. These textiles have embedded sensing capabilities and support a rich interaction space on and around the body. For example, StretchEBand [35] investigates how various stitching patterns of conductive thread can create piezoresistive textile sensors. Others have shown how smart textiles can augment musical performance [36], enhance prosthetic limbs [15], or support on-body interaction [27, 30, 33].

Our work complements these prior efforts by demonstrating ways to fabricate customized smart textiles with $3 \mathrm{D}$ printing and melt electrospinning. For example, we create a star-shaped capacitive sensor from rigid plastic and electrospun textile (Figure 8), and a liquid sensor that changes electrical resistance based on how much liquid is absorbed by an electrospun textile (Figure 10).

\section{Textiles and 3D Printing}

In the domain of 3D printing, textiles have been examined as a way to fabricate soft objects and explore the boundary between hard and flexible materials. Hudson [9] developed a 3D printer that prints needle-felted yarn, enabling the creation of new soft objects. Peng et al. [25] created a layered fabric printer that laser cuts sheets of fabric and adheres them together using heat sensitive adhesive.

Rather than directly printing textile materials, other work has explored combining existing textiles with traditional 3D 
Printing. Pei et al. [24] have shown it is possible to directly $3 \mathrm{D}$ print plastic onto textiles to create structures like a latch and hook. Others have explored lace- and knit-like structures using combinations of hard and flexible materials laid down using printed materials [21]. More recently, Rivera et al. [29] have demonstrated a range of techniques for embedding textiles into $3 \mathrm{D}$ printed objects during the printing process, opening a new design space of printed and flexible objects. Others have manipulated the softness of 3D printed objects by fabricating hair-like structures with rigid plastics [13, 23].

Our work differs from these prior efforts in that we focus on in-situ creation of textiles and rigid plastic objects in a single process (i.e. using a single base material from a single extruder). We modify a consumer-grade FDM printer to support fabricating textiles via melt electrospinning. Furthermore, unlike existing work that either embeds pre-fabricated textiles into plastic objects or vice versa, our printer can print both rigid plastic and textiles using a single plastic filament (PLA). This simplifies the printing process by no longer requiring users to insert textiles during the printing process or rigid material after a print is finished. Lastly, our work enables the placement of printed textiles to be digitally controlled, eliminating any alignment issues.

\section{Electrospinning}

Our work builds on efforts in the material and polymer science communities that have long explored using electrostatic forces to produce polymer fibers with small diameters (nanometer to micrometer scale). Electrostatic spinning, or electrospinning, is accomplished by applying a high electric potential to a polymer that is either melted, or dissolved into a solution using a solvent. The process has been used to create biological tissue scaffolding [34], protective clothing for agricultural workers [14], and even selectively permeable membranes [5]. We briefly describe aspects of electrospinning below. For a more comprehensive overview of electrospinning and its applications, we refer readers to these literature surveys $[2,8,11]$.

There are three major components to an electrospinning set-up: a high voltage power supply $(5-50 \mathrm{kV})$, a spinneret (typically a syringe needle) and a collector (a metal plate) $[2,8,11]$. In this work, we focus on supporting melt electrospinning alongside rigid plastic $3 \mathrm{D}$ printing using a single extruder. Using a solvent-based approach typically requires a ventilation system, which is not easily accessible nor wellsuited for typical consumer-grade 3D printer environments.

In melt electrospinning, a polymer is inserted into a spinneret and heated (e.g., with a heat gun or a hot circulating fluid). Once melted, a large charge differential is applied between the spinneret and the collector causing the melted material to be propelled out of the spinneret towards the collector (Figure 3).

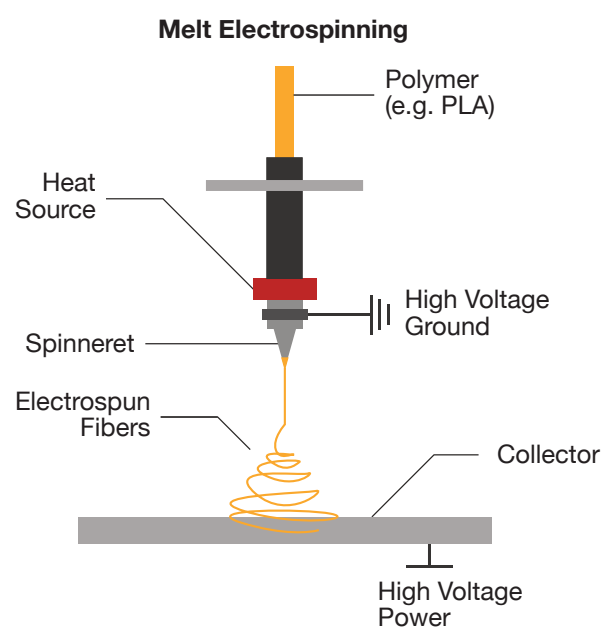

Figure 3: A typical melt electrospinning setup uses a heated polymer fed into a spinneret. A high electric potential is applied between spinneret tip and the metal collector to propel (typically micron-level) fibers down to the collector.

Recently, a sub-field of the melt electrospinning community has emerged that explores direct writing of fibers [4]. In direct writing, the spinneret and collector move to support controlled electrospinning. Our work is heavily influenced by these efforts. We support melt electrospinning on a consumer-grade 3D printer that has three axes of movement. However, we focus on producing both rigid plastic and electrospun fibers in the same process, expanding the range of objects that can be 3D printed.

Lastly, a former Kickstarter-based company, Electroloom ${ }^{1}$, demonstrated a consumer process for creating fabrics via solution electrospinning over custom 3D molds. Our process differs from theirs in that we use melt electrospinning which is safer than their solvent-based approach, and we print textiles and rigid plastic without the use of molds.

\section{ELECTROSPINNING 3D PRINTER CONSTRUCTION}

The supplied voltage in electrospinning can typically range between $5-50 \mathrm{kV}[2,8,11]$. Such a large electric potential introduces difficulties that can prevent a $3 \mathrm{D}$ printer from operating properly. Firstly, high voltage can cause electrical breakdown ${ }^{2}$ in which a material that is normally considered to be an electrical insulator (e.g., wire insulation or air) becomes conductive. Secondly, any voltage above the maximum rated voltage for a electronics can damage the electronics and cause malfunction during operation. Thirdly, high voltage can remain on the surface of a conductive material until it is

\footnotetext{
${ }^{1}$ https://www.kickstarter.com/projects/electroloom/electroloom-theworlds-first-3d-fabric-printer

${ }^{2}$ https://en.wikipedia.org/wiki/Electrical_breakdown
} 

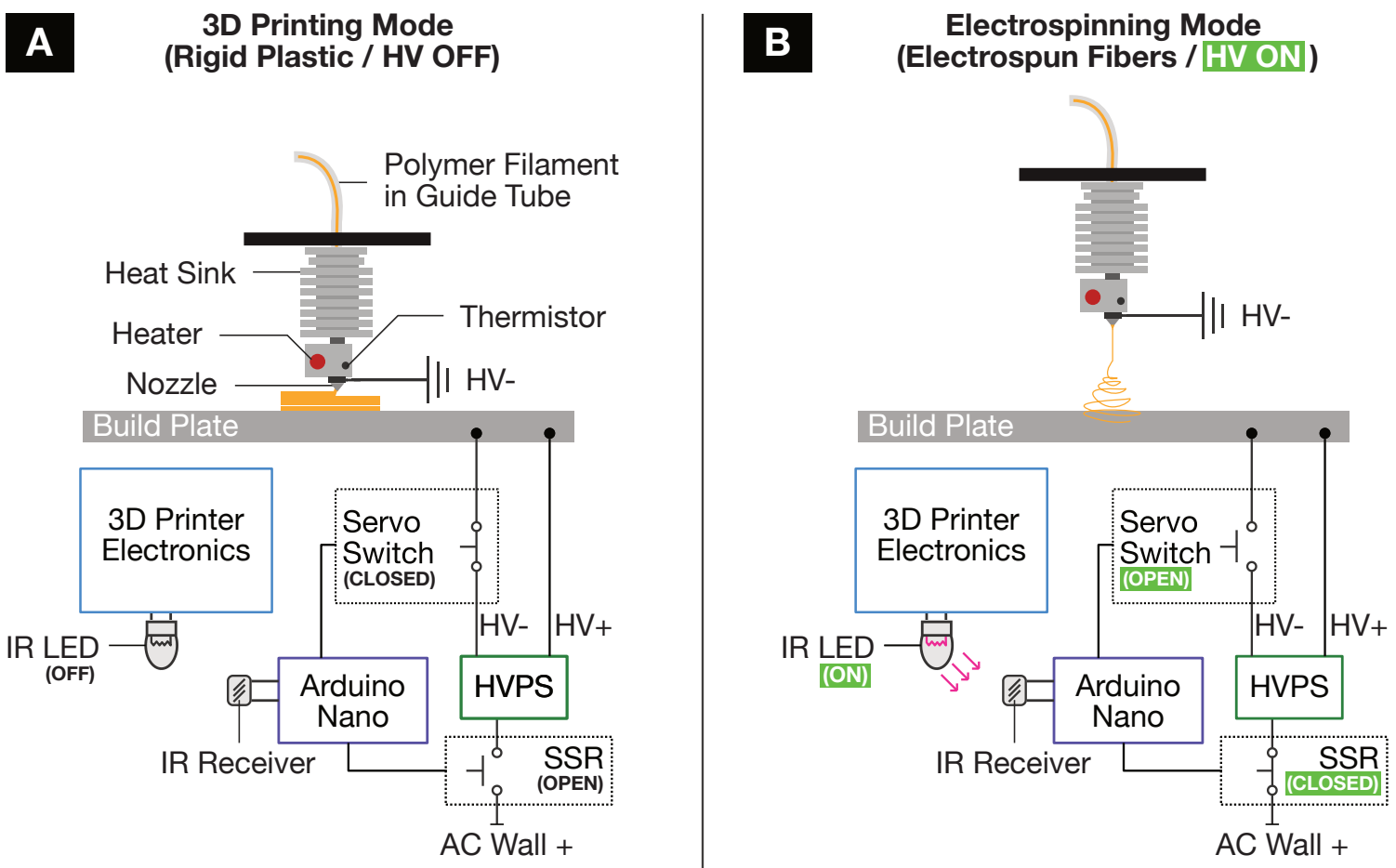

Figure 4: A simplified representation of how rigid plastic 3D printing and electrospinning are accomplished on our printer. In rigid plastic mode (A), the disabled IR LED triggers the build plate to be connected to the high voltage ground and the high voltage power supply (HVPS) is off. In electrospinning mode (B), the enabled IR LED triggers the build plate to be disconnected from the high voltage ground and the high voltage power supply is enabled.

discharged creating possible hazards for moving electronics (e.g., the hotend heater and thermistor).

Most consumer-grade 3D printers operate at comparatively low voltages in the range of $5-24 \mathrm{~V}$ (two orders of magnitude less than the applied voltage in melt electrospinning). Our printer also operates in this range using a typical $12 \mathrm{~V}$ power supply; we do not use any high voltage rated electronics apart from a high voltage power supply that is normally used in electrospinning. In the following section, we describe how we modified a consumer-grade 3D printer to support electrospinning by minimizing electrical breakdown, controlling electrical discharge, and isolating electronics.

Our printer is a variation of an open-source, consumergrade $3 \mathrm{D}$ printer ${ }^{3}$ modified to support melt electrospinning. We note that the hotend nozzle can be likened to the spinneret typically used in electrospinning, while the printer's metal (aluminum) build plate functions as the collector. We use a low current high voltage $(1 \mathrm{~mA} ; 5-35 \mathrm{kV})$ power supply ${ }^{4}$ (HVPS) to create an electrostatic potential between the hotend nozzle and build plate of the 3D printer (see Figure 4A) when we enable electrospinning during a print

\footnotetext{
${ }^{3}$ Similar to the Prusa I3: https://reprap.org/wiki/Prusa_i3

${ }^{4}$ https://www.amazing1.com/hv-dc-power-supplies.html
}

job. The HVPS is operated independently of the $12 \mathrm{~V}$ power supply that we use to operate the $3 \mathrm{D}$ printer's electronics.

Prior work on electrospinning has shown that either the spinneret or the collector can be wired with the positive voltage as long as the other is wired with the high voltage ground $[10,11,18,40]$. We experimented with both configurations and found that wiring the high voltage ground to the hotend nozzle and the high voltage positive wire to the build plate allowed us to more readily isolate the electronics and reduce potential charge being stored on the printer's frame during operation.

As a precaution, we wire the printer's frame to the high voltage ground to discharge any high voltage that manages to reach the frame. We note that while this high voltage can cause the electronics of the 3D printer to malfunction, the low current rating of our HVPS poses minimal risk for humans. Touching a surface charged by the HVPS only produces a zap sensation akin to a static shock.

In our initial investigations, we found that a minimum air gap of $1.5 \mathrm{~cm}$ is needed to prevent the applied high voltage $(7 \mathrm{kV})$ from arcing across to the high voltage ground while the HVPS is on. To control when the HVPS is enabled while limiting any potential high voltage interactions with the 


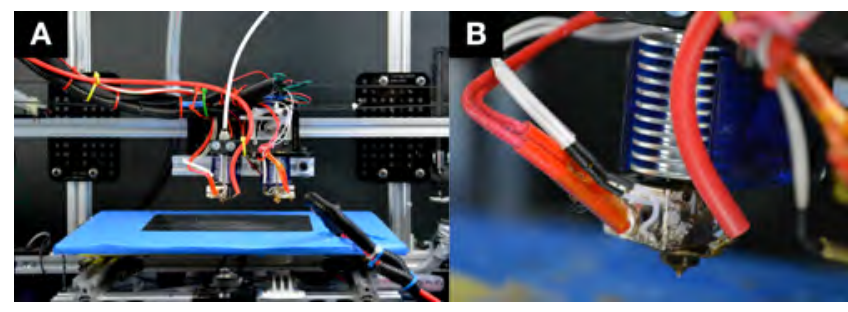

Figure 5: Our 3D printer that supports melt electrospinning and rigid plastic printing using only the left extruder (A). A close-up of the extruder shows the high voltage ground wire connected to hotend nozzle (B). We note that (A) shows an additional extruder on the right- a recent change for exploring a third material type (i.e. conductive filament).

3D printer electronics, we use a separate electrical circuit consisting of an Arduino Nano, a solid-state relay (SSR), an IR receiver, and a servo mechanism (as described below). The SSR is connected to the HVPS power input from a wall outlet (120 VAC). The signal of the SSR and the IR receiver are wired to the Arduino Nano. The HVPS's state is toggled when an IR LED signal sent from our printer's electronics is received by the Arduino Nano (Figure 4).

As mentioned before, high voltage charges can be retained in the surface of objects until discharged. Our initial explorations found the metal build plate would hold enough charge to shutdown the entire printer when switching between print modes. To prevent charge from being held on the printer's build plate during operation, we use the servo mechanism connected to the Arduino Nano to mechanically open or close a switch connecting the metal build plate to the high voltage ground. When the printer is in electrospinning mode, the servo opens the switch to disconnect the build plate from the high voltage ground (Figure 4B). When the printer is in normal plastic printing mode, the servo closes the switch to connect the build plate to the high voltage ground, discharging any remaining voltage (Figure 4A).

To ensure the servo motor is electrically isolated from the high voltage wires during operation, we maintain the minimum air gap distance between the servo motor, the high voltage wires and the build plate's contact terminals using a 3D printed plastic extension affixed to the servo. This ensures a safe distance is maintained for the wires and allows the contact terminals to touch without fear of arcing. The servo and SSR are operated by the Arduino Nano at the same time when the corresponding electrospinning mode on/off signal is received.

Because the hotend nozzle maintains a safe working distance while the HVPS is activated and any electric potential applied to the build plate is discharged once the HVPS is disabled, we are able to use a standard ceramic cartridge heater and thermistor to manage the temperature of the hotend during printing.

We note that we use an all-metal hotend (without PTFE inserts) ${ }^{5}$ to increase the range of print temperatures. Hotends with PTFE liners can experience melting of the liner when the temperature rises above $265^{\circ} \mathrm{C}$.

Lastly, we initially used a standard brass nozzle, however, we found that higher operation temperatures $\left(>270^{\circ} \mathrm{C}\right)$ during melt electrospinning caused the nozzle to wear more quickly. Our printer design now uses a $0.4 \mathrm{~mm}$ stainless steel hotend nozzle ${ }^{6}$. This modification is consistent with prior literature on melt electrospinning that has used stainless steel syringe tips for extrusion. With these modifications, we have successfully been able to operate our 3D printer at high temperatures to support melt electrospinning.

\section{D Printer Firmware}

We modified the printer's base firmware ${ }^{7}$ to handle operations that enable the electrospinning mode, namely sending a signal via IR LED and adjusting the extruder's $Z$ position offset to be at least the minimum air gap distance away from the build plate while electrospinning. When the normal plastic printing mode is enabled, the IR LED is disabled and the extruder's Z position offset returns to zero. Lastly, we support using a single extruder for both electrospinning and normal plastic printing by specifying there are two extruders in the firmware that share a heater and thermistor on a single hotend. We have open-sourced the printer's firmware ${ }^{8}$.

\section{D Printing of PLA and Electrospun PLA Fibers}

We primarily use Hatchbox PLA $1.75 \mathrm{~mm}$ filament ${ }^{9}$ in our $3 \mathrm{D}$ printer. We print rigid PLA at a temperature of $220^{\circ} \mathrm{C}$ with a combined movement and extrusion feed rate of 2400 $\mathrm{mm} / \mathrm{min}$. When printing electrospun fibers, we use the same material in same extruder, however, the temperature is much higher and feed rates are much slower. In general, we electrospin PLA at a temperature of $290^{\circ} \mathrm{C}$ with a movement feed rate $1300 \mathrm{~mm} / \mathrm{min}$, an extrusion feed rate of $10 \mathrm{~mm} / \mathrm{min}$ with objects processed at a $20 \%$ infill density. These parameters can be adjusted to achieve different effects. We describe these parameters and associated tests that we performed to determine them in the next section. G-code files generated for our 3D printer are post-processed with a Python script to ensure the proper feed rates for electrospinning are set before printing.

\footnotetext{
${ }^{5}$ https://www.amazon.com/Gulfcoast-Robotics-Extruder-PrinterFilament/dp/B07B4FHN72

${ }^{6} \mathrm{https}: / / \mathrm{www} . a m a z o n . c o m / I v e l i n k-S t a i n l e s s-E x t r u d e r-F i l a m e n t-$ Printer/dp/B077M8Z91C/

${ }^{7}$ https://github.com/repetier/Repetier-Firmware

${ }^{8}$ https://github.com/mriveralee/desktop-electrospinning/

${ }^{9}$ https://hatchbox3d.com/collections/pla/products/3d-pla-1kg1-75-gld
} 

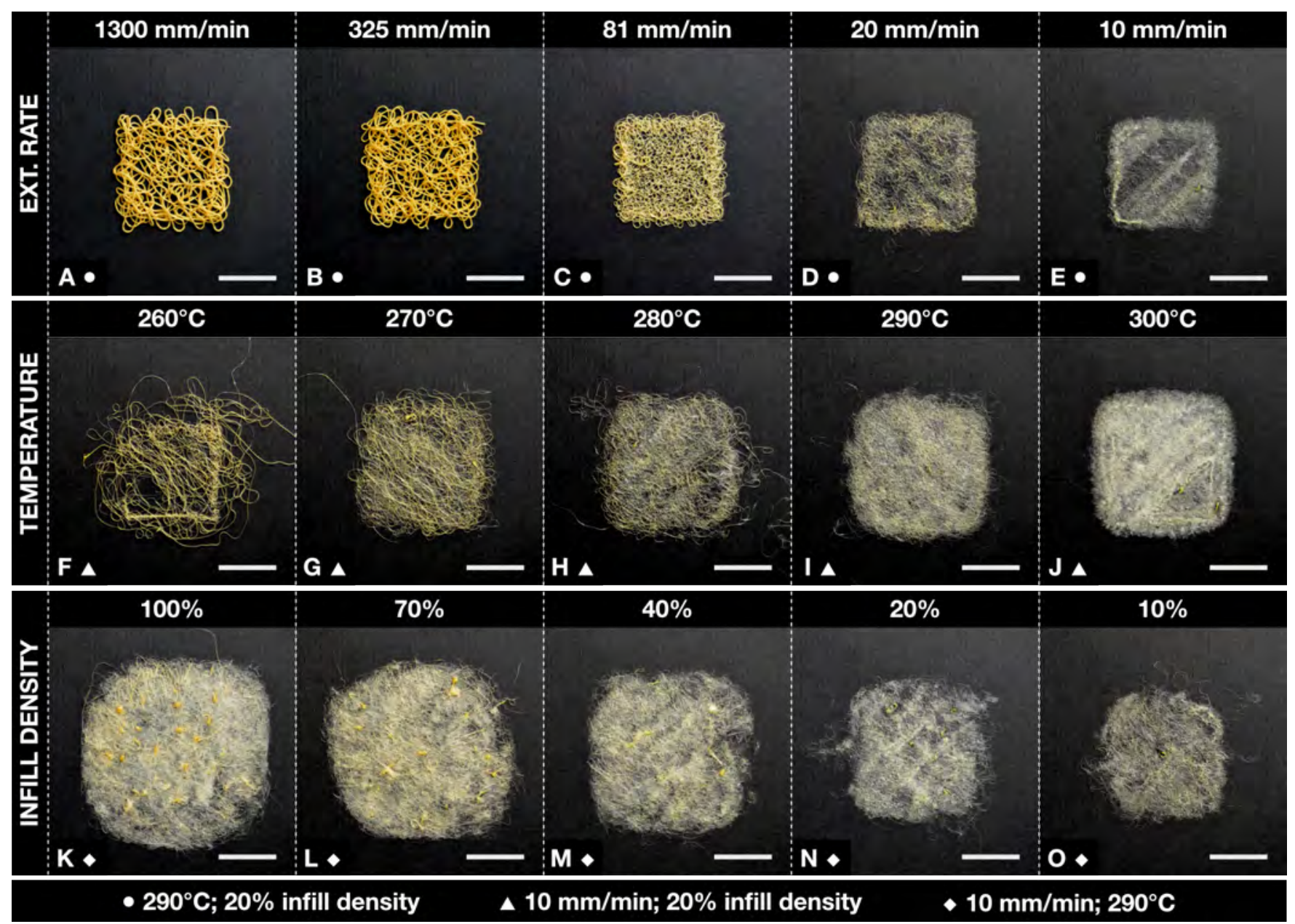

Figure 6: A series of tests we performed on extrusion rate (A-E), temperature (F-J), and infill density (K-O) to determine melt electrospinning process parameters for our 3D printer. Tool-paths were generated from the geometry of a 30x30mm square swatch using a 3D printer slicer engine. Scale bar: $20 \mathrm{~mm}$.

We represent the two different types of printing (electrospinning vs. normal) as separate configurations that may be combined in "dual" extrusion prints. Because electrospun fibers are spun at high temperatures and propelled down quickly, they readily bond once in contact with previously printed plastic. To ensure a stronger bond, one can print rigid plastic directly on top of desired regions of the fibers.

The material types may be printed in any order with two constraints: (1) the maximum height of rigid plastic that can be printed below regions that will have fibers spun on top is $0.6 \mathrm{~mm}$; (2) the maximum height of electrospun fibers is constrained to at most $1.2 \mathrm{~mm}$. We explain these constraints further in our Discussion and Limitations section.

Note that our printer can print these two material types using the same PLA filament on a single extruder without human-intervention during the printing process. Our firmware handles enabling high voltage and offsetting the extruder when necessary. The high voltage is set to $7 \mathrm{kV}$ and the extruder's $\mathrm{Z}$ position offset for electrospinning is set to $1.5 \mathrm{~cm}$ by default.

\section{ELECTROSPINNING PROCESS PARAMETERS}

In this section, we report the results of tests performed to determine optimal electrospinning parameters for our 3D printer (Figure 6). In agreement with prior work [11, 39], our results demonstrate temperature and extrusion rate are crucial factors for optimal electrospinning. We also describe how infill density ${ }^{10}$, a slicing parameter that controls material density, affects the size of an object when electrospun. Lastly, we discuss mitigating size discrepancies by applying a scale factor to the input geometry before generating

\footnotetext{
${ }^{10} \mathrm{https} / /$ ultimaker.com/en/resources/52670-infill
} 
A

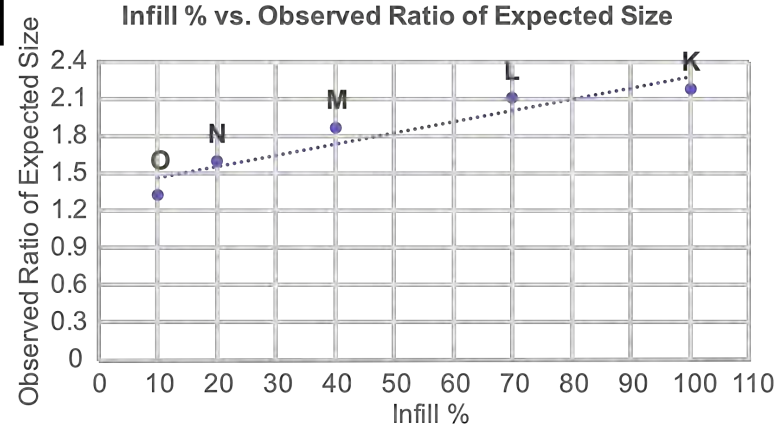

B

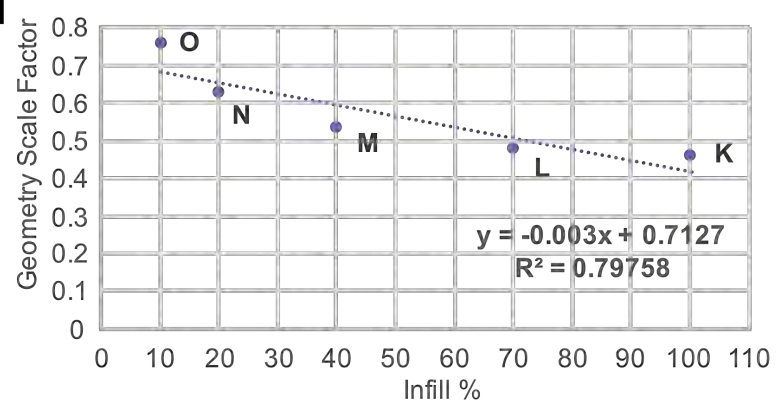

Figure 7: Infill density is positively correlated with the size of fabricated electrospun objects (A). The inverse of this relationship determines a geometry scale factor that when applied to objects prior to slicing will result in an electrospun object of desired size.

a printable tool-path. All the tests were performed using a $30 \times 30 \mathrm{~mm}$ square swatch with the same printing parameters except for the parameter being manipulated. The default parameters are an extrusion rate of $10 \mathrm{~mm} / \mathrm{min}$, a temperature of $290^{\circ} \mathrm{C}$, and a $20 \%$ infill density.

\section{Extrusion Rate}

Extrusion rate refers to the speed (and thereby amount) of material that is advanced during the printing process. For typical 3D printing, the feed rate (or movement speed for the $\mathrm{X}, \mathrm{Y}$, and $\mathrm{Z}$ axes in $\mathrm{mm} / \mathrm{min}$ ) along with hotend nozzle diameter, extrusion width, and layer height are used by the slicing engine to determine how much material should be advanced given a particular tool-path operation. For electrospinning, we found it is necessary to keep a slow, constant extrusion rate that is independent of the movement feed rate and these other parameters. As seen in Figure 6A-E, a fast extrusion rate (A) produces material that is more rigid and similar to typical 3D printed PLA. While a much slower extrusion rate (E) allows electrostatic force to pull out thin fibers creating a soft textile. To prevent inconsistent production of fibers and globs of material from forming at the nozzle, the extrusion rate must be held constant during the printing process.

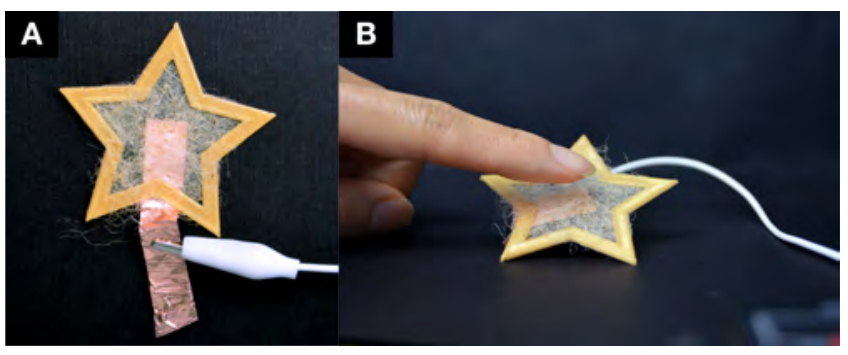

Figure 8: Electrospun fibers can be patterned onto conductive materials then bound together with rigid plastic to create custom-shaped capacitive sensors (A). The electrospun textile offers soft touch affordances and compliance to a capacitive sensor $(B)$.

\section{Temperature}

In agreement with prior research [11, 39], we found temperature affects the diameter of the produced electrospun fibers (Figure 6F-J). A low temperature of $260^{\circ} \mathrm{C}$ (though high compared to normal rigid plastic printing) causes fibers to be stringy and more plastic-like in appearance $(\mathrm{F})$. As the temperature increases, the diameter of produced fibers decreases. Anecdotally, we note that the fibers have diameters smaller than a typical human hair at $300^{\circ} \mathrm{C}(\mathrm{J})$. However, at this temperature and above, we found electrospinning became inconsistent- extrusion halted temporarily and plastic subsequently was extruded in the form of small beads.

\section{Infill Density}

We examined infill density, a common 3D printing slicing parameter, as a way to control material density in a tool-path for electrospinning. In our tests, we used a rectilinear infill pattern with the various fill densities listed in Figure 6K-O. We found infill density was positively correlated with the size of a produced electrospun object. In all cases, the infill density test tool-paths produced objects with dimensions larger than the expected 30x30mm square swatch. Figure 7A shows the relationship between infill density percentage and the ratio of the produced object's size to its expected size. To account for the size impact of a particular infill density on the produced electrospun object's size, we calculate a geometry scale factor as the inverse of this ratio as seen in Figure 7B. The geometry scale factor is applied to the size of 3D modeled objects prior to slicing.

We evaluated the geometry scale factor by printing 4 square electrospun swatches $(30 \times 30 \mathrm{~mm})$ at $20 \%$ infill with the scale factor of 0.65 (obtained using the fitted function in Figure 7B). We measured their width and height once fabricated. The average dimension across the swatches was 30.09 $\mathrm{mm}(\mathrm{SD}=0.49)$. In general, the geometry scale factor based on the infill density percentage is used to control the output 


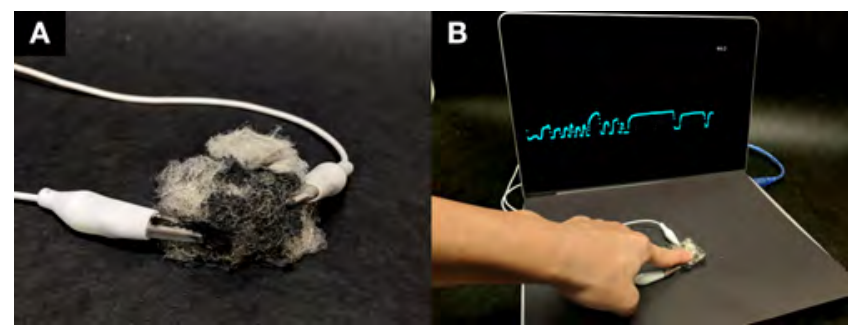

Figure 9: Electrospun fibers coated in conductive paint become a piezoresistive sensor (A), changing electrical resistance based on applied pressure (B).

shapes of our electrospun objects; however, because we are fabricating textiles, we can also post-process any deviations in size using similar textile-working techniques as [29].

\section{SOFT SENSOR FABRICATION}

Combining melt electrospinning with $3 \mathrm{D}$ printing affords new opportunities for creating soft sensors. In this section, we describe how we use electrospinning to facilitate sensing based on capacitance, piezoresistivity, and liquid absorption.

\section{Capacitive Sensing}

An electrospun textile is not conductive on its own. However, when combined with a conductor (e.g., copper foil), the textile can be used to create capacitive sensors that offer soft touch affordances. We control the placement of fibers to pattern custom-shaped sheets such as the star in Figure 8A onto a conductive material. We embed copper foil during the printing process after a layer of fibers is printed and the high voltage is disabled. We then continue printing with rigid plastic to create a binding enclosure for the copper foil and electrospun textile. The electrospun textile creates custom-shaped, soft affordances for the capacitive sensor while serving as a compliant material that mediates changes in capacitance Figure 8B.

\section{Piezoresistive Sensing}

Electrospun swatches or collections of fibers can be made piezoresistive by coating them in a mixture of conductive paint (Figure 9A). We mix a 1:2 ratio of conductive paint to water by volume and coat areas of the electrospun textile using a paint brush. The sensors are left to dry overnight. After attaching test leads to the ends of the sensing region, manipulation of the textile produces changes in electrical resistivity (Figure 9B).

\section{Liquid Absorption Sensing}

Fibers produced via melt electrospinning can be used to absorb liquids. We use this property to create an absorption sensor that changes electrical resistance based on how much

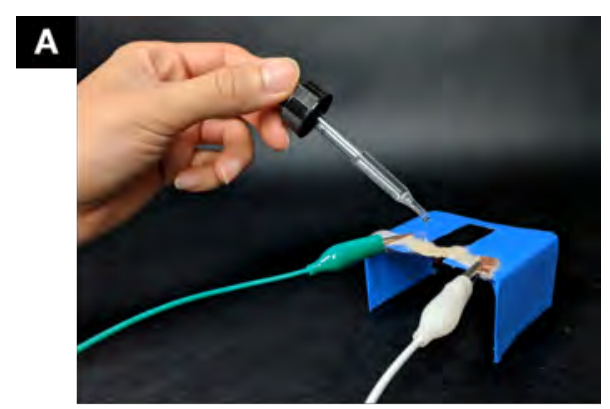

B

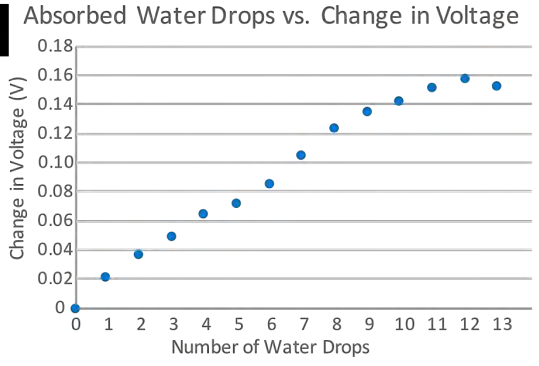

Figure 10: Experimental setup (A) and results (B) for evaluating electrospun fibers as a liquid absorption sensor. Each additional drop of water added to the fibers causes an increase in the sensed voltage between the two electrodes fixed at opposite ends of the fibers.

liquid is absorbed. We tested the feasibility of this sensor by attaching test leads to copper electrodes at opposite ends of a folded electrospun swatch (fabricated as a 30x30mm square).

Initially there is no electrical connection formed as there is no fluid to carry charge across the fibers. We place the swatch in tap water to establish an initial connection and then squeeze the swatch to remove as much water as possible. The swatch is placed in the test setup seen in Figure 10A. We then use a liquid dropper $(1 \mathrm{ml})$ to add individual drops of water to the center of the electrospun fibers.

After each additional water drop, we wait approximately 15 seconds for the voltage reading to stabilize then record the value. The chart in Figure 10B shows that increasing the amount of water in the fibers causes an increase in the sensed voltage (or decrease in electrical resistance) between the two electrodes fixed at the ends of the fibers. The 13th drop of water results in the fibers becoming overly saturated and expelling some water. This loss of water leads to a lower sensed voltage.

\section{APPLICATIONS}

We created a set of examples to demonstrate various looks, feels and interactions (e.g., water absorption sensing) enabled with our printer. For each example, we describe the electrospinning process parameters and their intended effects. 


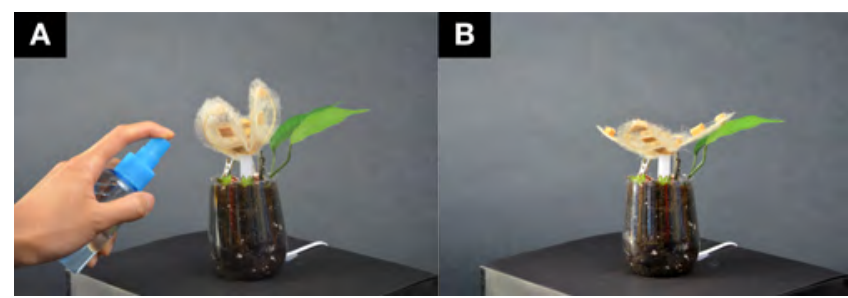

Figure 11: A custom-shaped flower made of electrospun textile and rigid plastic that actuates based on the soil's water level sensed using an electrospun liquid absorption sensor. When the soil is dry, the flower closes it petals (A). When the soil is moist, the flower actuates open.

\section{Water Me: Actuated Flower Reminder}

We fabricated a flower with soft electrospun petals and rigid plastic channels that enable tendon actuation with gel-spun polyethylene fishing line. The flower is electrospun with a mid-infill density, high temperature, and low extrusion rate $\left(40 \% ; 290^{\circ} \mathrm{C} ; 11 \mathrm{~mm} / \mathrm{min}\right)$, creating a soft, dense surface for supporting the rigid plastic channels. In addition, we electrospun a sensor that changes electrical resistance based on the amount of moisture absorbed in its fibers. Together, these objects are placed in a flower pot alongside a living plant to provide visual and physical feedback of the soil's moisture level. When the sensor detects the soil is dry, the flower's petals close using a servo motor (Figure 11A). Once water is added to the soil, the servo releases and the flower's petals open, indicating sufficient water is available for the nearby living plants (Figure 11B).

\section{Interactive Wooly the Sheep Comic}

We created a touch-sensitive comic of a sheep named "Wooly" using paper, copper foil traces and a custom-shaped electrospun textile. We fabricated the comic by inkjet printing the comic onto paper, adding a copper foil trace for wiring connections, applying light adhesive using a glue stick and then electrospinning directly onto the comic. The body of the sheep is electrospun with a high infill density, a mid-temperature, and a mid-extrusion rate $\left(50 \% ; 280^{\circ} \mathrm{C} ; 18\right.$ $\mathrm{mm} / \mathrm{min}$ ) to achieve a fluffy and spongy tactile experience. Capacitive sensors are embedded under the sheep's electrospun fibers: one sensor is present on the sheep's back (visible in Figure 12A for the purposes of showing the sensor); the other is placed on the sheep's head behind the comic paper. When the sheep's back is petted, a "Baaah" sound characteristic of a sheep is produced. When the sheep's head is touched, a light-hearted giggle is produced to indicate the sheep's enjoyment. This example demonstrates the possibility of using existing objects (i.e. paper and copper foil) alongside electrospun fibers to create soft, custom-shaped interactive experiences.

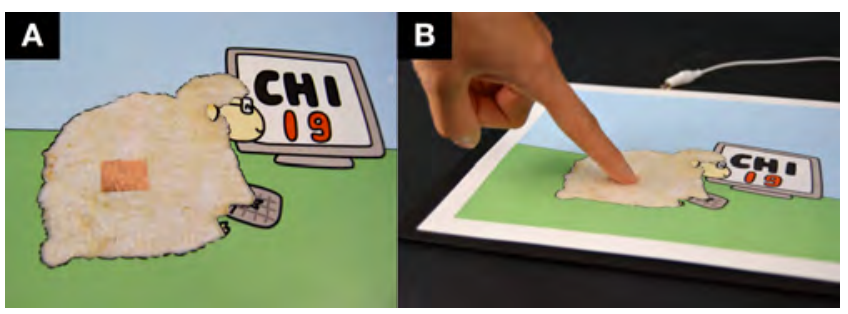

Figure 12: An interactive comic featuring a cartoon sheep whose body is made of a custom-shaped electrospun textile for soft capacitive sensing and tactile experience (A). Touching the sheep in various regions of its body produces different sound effects from a "Baaaa" to a giggle (B).

\section{Foldaway Phone Stand}

We printed a flexible, foldable stand that can be affixed to a mobile phone to offer a preferred viewing angle for watching videos (Figure 13A). The stand features a custom-shaped electrospun textile fabricated with a low infill density, high temperature, and low extrusion rate $\left(20 \% ; 298^{\circ} \mathrm{C} ; 10 \mathrm{~mm} / \mathrm{min}\right)$ to create a smooth and soft surface for hinging. Rigid plastic patterned on the textile enables bending into a configuration that can support the weight of the accompanying phone (Figure 13B). When not in use, the stand can be flattened to minimize its size. Anecdotally, the stand was used approximately 30 times to watch videos before the folded regions began to wear down and separate. We believe this issue could be mitigated by adding more layers of electrospun textile beyond the four used to fabricate the object.

\section{Textile Origami Lamp and Controls}

We fabricated an origami-style lamp that is printed flat using rigid plastic and electrospun textile (Figure 14A). The lamp's electrospun areas have a low infill density, high temperature, and low extrusion rate $\left(10 \% ; 290^{\circ} \mathrm{C} ; 10 \mathrm{~mm} / \mathrm{min}\right)$ to create a surface that diffuses the lamp's light. The lamp takes on a 3D form when folded and placed into its accompanying 3D printed holder. In addition, we printed a capsule that holds electrospun piezoresistive fibers (Figure 14B) and use this sensor to control the lamp's brightness (Figure 1B). Lastly, the lamp can be switched on and off using a soft, star-shaped capacitive toggle switch.

\section{DISCUSSION AND LIMITATIONS}

There are a few limitations of our approach to fabricating combined rigid plastic and electrospun textile-based objects. First, the height of electrospun textiles produced on the printer is constrained by the strength of the electric field between the hotend nozzle and the printer surface. As more non-conductive material is printed or placed between the two charged surfaces, the electric field strength decreases, 


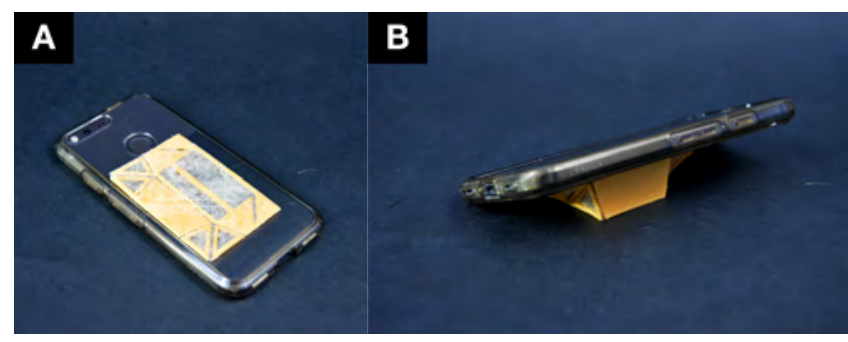

Figure 13: A foldaway phone stand fabricated using rigid plastic and electrospun textile. The phone stand is affixed to a phone case (A) and can be deployed when needed to obtain a preferred view angle for watching videos.

reducing the ability to electrospin. In line with existing work on melt electrospinning, we are only able to electrospin textiles that have heights of at most $1.2 \mathrm{~mm}$. We plan to explore using conductive materials (e.g., conductive paint, and conductive PLA) during the printing process to enable printing larger heights of electrospun textiles alongside rigid plastic.

Similarly, when printing large plastic objects (e.g., a tower), because the height of the extruder used for electrospinning must be raised to prevent collisions, the ability to electrospin is also reduced. This is a result of the electric field strength being inversely proportional to the squared distance between the two charged surfaces (the hotend nozzle and the build plate). On our printer, we found the minimum distance for electrospinning at $7 \mathrm{kV}$ without electrical arcing is $15 \mathrm{~mm}$ while the maximum distance before the electric field strength becomes too weak to electrospin is $50 \mathrm{~mm}$. We are exploring ways to increase this range such as adjusting the applied high voltage dynamically as a function of the current printing height and using a positionable charged probe for targeted electrospinning similar to collector probe arrays in [17].

\section{FUTURE WORK}

There are many interesting opportunities for future work in addition to addressing limitations of our approach for creating mixed soft and rigid objects on a 3D printer. The material science community has seen other polymers electrospun such as thermoplastic polyurethane (TPU) and polycaprolactone (PCL) [11], we are currently experimenting with these materials to extend the range of objects we can fabricate.

We would also like to explore selectively applying high voltage to areas of the build plate using a grid system to control the direction and orientation of electrospun fibers. This approach could allow for creating interesting textilebased displays that present different information depending on how the fibers are oriented. Additionally, we would like to explore dying and coating fibers in different substances to create unique material properties post-printing. For example, hydrophobic coatings could enable objects to repel water.

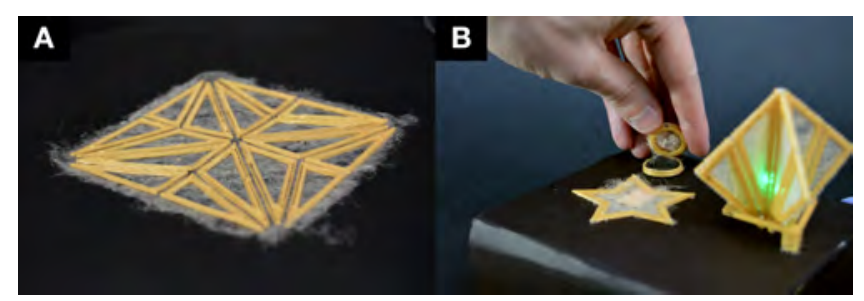

Figure 14: A custom-shaped origami-style lamp printed using rigid plastic and electrospun textile (A). Once folded into its final form, the lamp is paired with a soft, star-shaped capacitive toggle switch, and an electrospun piezoresistive capsule sensor to control the lamp's brightness (B).

Another related line of inquiry is focused on selective absorption of liquids. Recent work in the polymer science community has shown melt-electrospun PLA is effective at separating oil from water and may be useful in treating marine oil spill pollution [16]. We intend to explore how to create electrospun textiles that are optimized to absorb different liquids for interactive purposes. We would also like to investigate the potential environmental impact of using our approach to separate contaminants from water and air.

Lastly, we are interested in tackling sustainability issues using our process. Prior work has demonstrated melt electrospinning can be accomplished with recycled materials (e.g., PET bottles) [28]. We are examining how PLA from failed prints or disposed parts can be recycled into a melt electrospinning material for our 3D printer.

\section{CONCLUSION}

We have presented an approach to fabricating objects made of rigid plastic and electrospun textiles in a single process using a single extruder on 3D printer that supports melt electrospinning. We have demonstrated a range of objects that can be created using our method, from an interactive comic, to a flower that actuates based on sensed water levels. We have also shown how properties of electrospun textiles such as absorbency and softness can be leveraged to create customized soft sensors.

Our consumer-grade 3D printer is equipped with low-cost modifications to support melt electrospinning that make it amenable for desktop usage in the home. We believe the incorporation of melt electrospinning into current fabrication processes (i.e. 3D printing) opens interesting opportunities for the $\mathrm{HCI}$ community to explore 3D printing of interactive objects that blend rigidity and softness. Lastly, our approach introduces a new perspective from the material and polymer science communities that can broaden impact and thread new opportunities for the application of HCI. 


\section{ACKNOWLEDGMENTS}

We would like to acknowledge Siyan Zhao for her endless support and help with documenting the applications, and Queenie Kravitz for her support and inspiration. This work was funded in part by the National Science Foundation under grant IIS-1718651.

\section{REFERENCES}

[1] Moritz Bächer, Benjamin Hepp, Fabrizio Pece, Paul G. Kry, Bernd Bickel, Bernhard Thomaszewski, and Otmar Hilliges. 2016. DefSense: Computational Design of Customized Deformable Input Devices. In Proceedings of the 2016 CHI Conference on Human Factors in Computing Systems (CHI '16). ACM, New York, NY, USA, 3806-3816. https://doi. org/10.1145/2858036.2858354

[2] Nandana Bhardwaj and Subhas C Kundu. 2010. Electrospinning: a fascinating fiber fabrication technique. Biotechnology advances 28, 3 (2010), 325-347.

[3] Bernd Bickel, Moritz Bächer, Miguel A. Otaduy, Hyunho Richard Lee, Hanspeter Pfister, Markus Gross, and Wojciech Matusik. 2010. Design and Fabrication of Materials with Desired Deformation Behavior. In ACM SIGGRAPH 2010 Papers (SIGGRAPH '10). ACM, New York, NY, USA, Article 63, 10 pages. https://doi.org/10.1145/1833349.1778800

[4] Toby D Brown, Paul D Dalton, and Dietmar W Hutmacher. 2011. Direct writing by way of melt electrospinning. Advanced Materials 23, 47 (2011), 5651-5657.

[5] PW Gibson, HL Schreuder-Gibson, and D Rivin. 1999. Electrospun fiber mats: transport properties. AIChE journal 45, 1 (1999), 190-195.

[6] Kathleen Hajash, Bjorn Sparrman, Christophe Guberan, Jared Laucks, and Skylar Tibbits. 2017. Large-Scale Rapid Liquid Printing. 3D Printing and Additive Manufacturing 4, 3 (2017), 123-132.

[7] Thomas J Hinton, Quentin Jallerat, Rachelle N Palchesko, Joon Hyung Park, Martin S Grodzicki, Hao-Jan Shue, Mohamed H Ramadan, Andrew R Hudson, and Adam W Feinberg. 2015. Three-dimensional printing of complex biological structures by freeform reversible embedding of suspended hydrogels. Science advances 1, 9 (2015), e1500758.

[8] Zheng-Ming Huang, Y-Z Zhang, M Kotaki, and S Ramakrishna. 2003. A review on polymer nanofibers by electrospinning and their applications in nanocomposites. Composites science and technology 63, 15 (2003), 2223-2253.

[9] Scott E. Hudson. 2014. Printing Teddy Bears: A Technique for 3D Printing of Soft Interactive Objects. In Proceedings of the SIGCHI Conference on Human Factors in Computing Systems (CHI '14). ACM, New York, NY, USA, 459-468. https://doi.org/10.1145/2556288.2557338

[10] Matthew T Hunley, Afia S Karikari, Matthew G McKee, Brian D Mather, John M Layman, Ann R Fornof, and Timothy E Long. 2008. Taking advantage of tailored electrostatics and complementary hydrogen bonding in the design of nanostructures for biomedical applications. In Macromolecular symposia, Vol. 270. Wiley Online Library, 1-7.

[11] Dietmar W Hutmacher and Paul D Dalton. 2011. Melt electrospinning. Chemistry-An Asian fournal 6, 1 (2011), 44-56.

[12] Alexandra Ion, Johannes Frohnhofen, Ludwig Wall, Robert Kovacs, Mirela Alistar, Jack Lindsay, Pedro Lopes, Hsiang-Ting Chen, and Patrick Baudisch. 2016. Metamaterial Mechanisms. In Proceedings of the 29th Annual Symposium on User Interface Software and Technology (UIST '16). ACM, New York, NY, USA, 529-539. https://doi.org/10. 1145/2984511.2984540

[13] Gierad Laput, Xiang 'Anthony' Chen, and Chris Harrison. 2015. 3D Printed Hair: Fused Deposition Modeling of Soft Strands, Fibers, and Bristles. In Proceedings of the 28th Annual ACM Symposium on User Interface Software \&\#38; Technology (UIST '15). ACM, New York, NY,
USA, 593-597. https://doi.org/10.1145/2807442.2807484

[14] Seungsin Lee and S Kay Obendorf. 2006. Developing protective textile materials as barriers to liquid penetration using melt-electrospinning. Journal of Applied Polymer Science 102, 4 (2006), 3430-3437.

[15] Joanne Leong, Patrick Parzer, Florian Perteneder, Teo Babic, Christian Rendl, Anita Vogl, Hubert Egger, Alex Olwal, and Michael Haller. 2016. proCover: Sensory Augmentation of Prosthetic Limbs Using Smart Textile Covers. In Proceedings of the 29th Annual Symposium on User Interface Software and Technology, UIST 2016, Tokyo, Japan, October 16-19, 2016. 335-346. https://doi.org/10.1145/2984511.2984572

[16] Haoyi Li, Yi Li, Weimin Yang, Lisheng Cheng, and Jing Tan. 2017. Needleless Melt-Electrospinning of Biodegradable Poly (Lactic Acid) Ultrafine Fibers for the Removal of Oil from Water. Polymers 9, 2 (2017), 3.

[17] Yifang Liu, Ruimin Liu, Xiang Wang, Jiaxin Jiang, Wenwang Li, Juan Liu, Shumin Guo, and Gaofeng Zheng. 2018. Electrospun ThreeDimensional Nanofibrous Structure via Probe Arrays Inducing. Micromachines 9, 9 (Aug 2018), 427. https://doi.org/10.3390/mi9090427

[18] Jason Lyons, Christopher Li, and Frank Ko. 2004. Melt-electrospinning part I: processing parameters and geometric properties. Polymer 45, 22 (2004), 7597-7603.

[19] Jonàs Martínez, Jérémie Dumas, and Sylvain Lefebvre. 2016. Procedural Voronoi Foams for Additive Manufacturing. ACM Trans. Graph. 35, 4, Article 44 (July 2016), 12 pages. https://doi.org/10.1145/2897824. 2925922

[20] Vittorio Megaro, Jonas Zehnder, Moritz Bächer, Stelian Coros, Markus Gross, and Bernhard Thomaszewski. 2017. A Computational Design Tool for Compliant Mechanisms. ACM Trans. Graph. 36, 4, Article 82 (July 2017), 12 pages. https://doi.org/10.1145/3072959.3073636

[21] R Melnikova, A Ehrmann, and K Finsterbusch. 2014. 3D printing of textile-based structures by Fused Deposition Modelling (FDM) with different polymer materials. In IOP Conference Series: Materials Science and Engineering, Vol. 62. IOP Publishing, 012018.

[22] Satoshi Nakamaru, Ryosuke Nakayama, Ryuma Niiyama, and Yasuaki Kakehi. 2017. FoamSense: Design of Three Dimensional Soft Sensors with Porous Materials. In Proceedings of the 30th Annual ACM Symposium on User Interface Software and Technology, UIST 2017, Quebec City, QC, Canada, October 22 - 25, 2017. 437-447. https: //doi.org/10.1145/3126594.3126666

[23] Jifei Ou, Gershon Dublon, Chin-Yi Cheng, Felix Heibeck, Karl Willis, and Hiroshi Ishii. 2016. Cilllia: 3D Printed Micro-Pillar Structures for Surface Texture, Actuation and Sensing. In Proceedings of the 2016 CHI Conference on Human Factors in Computing Systems (CHI '16). ACM, New York, NY, USA, 5753-5764. https://doi.org/10.1145/2858036. 2858257

[24] Eujin Pei, Jinsong Shen, and Jennifer Watling. 2015. Direct 3D printing of polymers onto textiles: experimental studies and applications. Rapid Prototyping fournal 21, 5 (2015), 556-571.

[25] Huaishu Peng, Jennifer Mankoff, Scott E. Hudson, and James McCann. 2015. A Layered Fabric 3D Printer for Soft Interactive Objects. In Proceedings of the 33rd Annual ACM Conference on Human Factors in Computing Systems (CHI '15). ACM, New York, NY, USA, 1789-1798. https://doi.org/10.1145/2702123.2702327

[26] Jesús Pérez, Miguel A. Otaduy, and Bernhard Thomaszewski. 2017. Computational Design and Automated Fabrication of Kirchhoffplateau Surfaces. ACM Trans. Graph. 36, 4, Article 62 (July 2017), 12 pages. https://doi.org/10.1145/3072959.3073695

[27] Ivan Poupyrev, Nan-Wei Gong, Shiho Fukuhara, Mustafa Emre Karagozler, Carsten Schwesig, and Karen E. Robinson. 2016. Project Jacquard: Interactive Digital Textiles at Scale. In Proceedings of the $2016 \mathrm{CHI}$ Conference on Human Factors in Computing Systems, San fose, CA, USA, May 7-12, 2016. 4216-4227. https://doi.org/10.1145/2858036.2858176 
[28] H Rajabinejad, R Khajavi, A Rashidi, N Mansouri, and ME Yazdanshenas. 2009. Recycling of used bottle grade poly ethyleneterephthalate to nanofibers by melt-electrospinning method. Int. f. Environ. Res 3, 4 (2009), 663-670.

[29] Michael L. Rivera, Melissa Moukperian, Daniel Ashbrook, Jennifer Mankoff, and Scott E. Hudson. 2017. Stretching the Bounds of 3D Printing with Embedded Textiles. In Proceedings of the 2017 CHI Conference on Human Factors in Computing Systems (CHI '17). ACM, New York, NY, USA, 497-508. https://doi.org/10.1145/3025453.3025460

[30] T. Scott Saponas, Chris Harrison, and Hrvoje Benko. 2011. PocketTouch: Through-fabric Capacitive Touch Input. In Proceedings of the 24th Annual ACM Symposium on User Interface Software and Technology (UIST '11). ACM, New York, NY, USA, 303-308. https: //doi.org/10.1145/2047196.2047235

[31] Martin Schmitz, Jürgen Steimle, Jochen Huber, Niloofar Dezfuli, and Max Mühlhäuser. 2017. Flexibles: Deformation-Aware 3D-Printed Tangibles for Capacitive Touchscreens. In Proceedings of the 2017 CHI Conference on Human Factors in Computing Systems (CHI '17). ACM, New York, NY, USA, 1001-1014. https://doi.org/10.1145/3025453.3025663

[32] Christian Schumacher, Bernd Bickel, Jan Rys, Steve Marschner, Chiara Daraio, and Markus Gross. 2015. Microstructures to Control Elasticity in 3D Printing. ACM Trans. Graph. 34, 4, Article 136 (July 2015), 13 pages. https://doi.org/10.1145/2766926

[33] Julia Schwarz, Chris Harrison, Scott Hudson, and Jennifer Mankoff 2010. Cord Input: An Intuitive, High-accuracy, Multi-degree-offreedom Input Method for Mobile Devices. In Proceedings of the SIGCHI Conference on Human Factors in Computing Systems (CHI '10). ACM, New York, NY, USA, 1657-1660. https://doi.org/10.1145/1753326. 1753573

[34] J Venugopal and S Ramakrishna. 2005. Applications of polymer nanofibers in biomedicine and biotechnology. Applied biochemistry and biotechnology 125, 3 (2005), 147-157.

[35] Anita Vogl, Patrick Parzer, Teo Babic, Joanne Leong, Alex Olwal, and Michael Haller. 2017. StretchEBand: Enabling Fabric-based Interactions Through Rapid Fabrication of Textile Stretch Sensors. In Proceedings of the 2017 CHI Conference on Human Factors in Computing Systems (CHI '17). ACM, New York, NY, USA, 2617-2627. https://doi.org/10. $1145 / 3025453.3025938$

[36] Irmandy Wicaksono and Joseph A. Paradiso. 2017. Fabrickeyboard: multimodal textile sensate media as an expressive and deformable musical interface. In 17th International Conference on New Interfaces for Musical Expression, NIME 2017, Aalborg University, Copenhagen, Denmark, May 15-18, 2017. 348-353. http://www.nime.org/proceedings/ 2017/nime2017_paper0066.pdf

[37] Sang Ho Yoon, Ke Huo, Yunbo Zhang, Guiming Chen, Luis Paredes, Subramanian Chidambaram, and Karthik Ramani. 2017. iSoft: A Customizable Soft Sensor with Real-time Continuous Contact and Stretching Sensing. In Proceedings of the 30th Annual ACM Symposium on User Interface Software and Technology (UIST '17). ACM, New York, NY, USA, 665-678. https://doi.org/10.1145/3126594.3126654

[38] Jonas Zehnder, Espen Knoop, Moritz Bächer, and Bernhard Thomaszewski. 2017. Metasilicone: Design and Fabrication of Composite Silicone with Desired Mechanical Properties. ACM Trans. Graph. 36, 6, Article 240 (Nov. 2017), 13 pages. https://doi.org/10.1145/3130800. 3130881

[39] Li-Hua Zhang, Xiao-Peng Duan, Xu Yan, Miao Yu, Xin Ning, Yong Zhao, and Yun-Ze Long. 2016. Recent advances in melt electrospinning. RSC Advances 6, 58 (2016), 53400-53414.

[40] Eduard Zhmayev, Daehwan Cho, and Yong Lak Joo. 2010. Modeling of melt electrospinning for semi-crystalline polymers. Polymer 51, 1 (2010), 274-290. 